\title{
Multiobjective Optimization of Fuzzy Logic Scheduled Controllers for Missile Autopilot Design
}

\author{
Anna Blumel, Brian White \\ Department of Aerospace, Power and Sensors, Cranfield University \\ Shrivenham, Wilts, SN6 8LA, UK \\ A.L.Blumel@rmcs.cranfield.ac.uk, B.A.White@rmcs.cranfield.ac.uk
}

\begin{abstract}
This paper describes the design of a Fuzzy Logic Scheduled Controllers used for side-slip velocity control of a missile autopilot design. A multiobjective evolutionary algorithm is used to determine the membership function distribution of the fuzzy trajectory controller within an outer loop control system. Scaling factors of the FLC inputs and outputs for each required demand are obtained by using a polynomial fit for a large range of multiple velocity demands $(1 \mathrm{~g}, 5 \mathrm{~g}, 10 \mathrm{~g}, 15 \mathrm{~g}$-lateral acceleration equivalent). The design meets mulitple objectives related to closed loop performance such as: steady state error, overshoot, settling and rise time. Multiple solutions are obtained simultaneously by using nondominated sorting for forming the Pareto front, combined with a reference point approach to incorporate preference information into the GA to direct the search towards feasible desirable areas which satisfy specific values of the objectives.Simulationn results are presented showing the fuzzy gain surface and extreme models in the multip[le model population.
\end{abstract}

\section{Introduction}

Many problems involve simultaneous optimization of multiple objectives and the design of a missile autopilot is one such application area. One of the main requirements for an autopilot design is to yield a fast response with the minimum of overshoot with good accuracy. Fast rise time or settling time can measure the speed of response, and overshoot and steady state level can then complete the measurement of the time response. These measurements or objective functions will produce conflicting requirements as fast responses tend to produce overshoot and damped responses slow down the speed of response. Good steady state error performance usually demands high gains or the inclusion of integrators.n Both of these will produce less stable solutions that have significant overshoot. Hence there is a need to determine the trade-off mechanism for such conflicting requirements and to provide a method for designing such systems. This implies that any method of tuning the trajectory control parameters should consider the four objectives simultaneously: the usual single-objective optimization problem has become multi-objective optimization. This will produce multiple solutions rather than a single solution associated with a single objective. There is also considerable uncertainty in the dyanmics associated with the missile airframe which has to be taken into account in the design process. In previous research [1] a combination of an input/output linearisation technique (nonlinear control law) and a fuzzy logic trajectory controller has been considered solving the scalar optimization problem. This format is retained but the fuzzy logic trajectory controller is now designed using the multiple objective approach.

The paper will look at designing an autopilot for a highly nonlinear missile over large ranges of incidence. The technique will obtain multiple solutions for sideslip velocity control of the missile for a large range of multiple demands, thus exercising the nonlinear model over its entire range. Side-slip velocity demands are considered for both pitch and yaw planes, using the missile rudder and elevator as control surfaces hence yielding a system with 2 inputs and 2 controlled outputs. The autopilot design uses an evolutionary algorithm optimisation approach to a multiple model description of the airframe aerodynamics. This is used to determine the membership function distribution within the outer fuzzy loop control system by using a multi-objective evolutionary algorithm that meets objectives related to closed loop performance such as: rising and settling time, steady state error, and overshoot. Fuzzy scheduled controllers having scaling factors on both inputs and outputs by using polynomial fit to allow for a large range of required demands. 


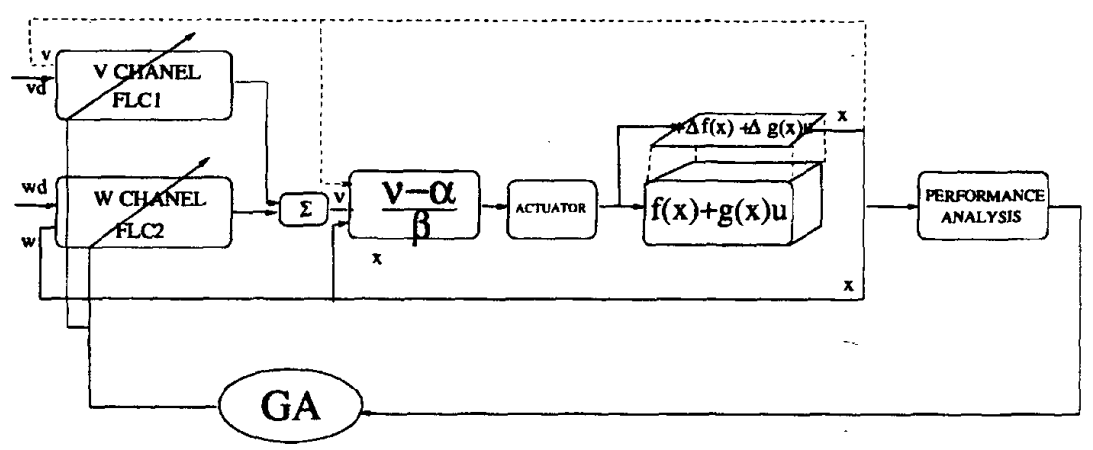

Figure 1. Trajectory control design

\section{Fuzzy Autopilot Design}

The autopilot design process is shown in figure 1. The autopilot consists of an inner linearisation loop, together with an out fuzzy controller to improve the robustness of the design. The missile dynamics are given by:

$$
\begin{aligned}
\dot{x} & =f(x)+\Delta f(x)+(g(x)+\Delta g(x)) u \\
y & =h(x)
\end{aligned}
$$

which are non-linear and have significant uncertainty $\Delta f(x)$ and $\Delta g(x)$. The description of the model is obtained from data supplied by MatraBAE and detailed in the Horton report [2].

The angular and translational equations of motion of the missile airframe are given by:

$$
\begin{aligned}
\dot{q} & =\frac{1}{2} I_{y z}^{-1} \rho V_{o} S d\left(\frac{1}{2} d C_{m q} q+C_{m w} w+V_{o} C_{m \eta} \eta\right) \\
\dot{w} & =\frac{1}{2 m} \rho V_{o} S\left(C_{z w} w+V_{o} C_{z \eta} \eta\right)+U q \\
\dot{r} & =\frac{1}{2} I_{y z}^{-1} \rho V_{o} S d\left(\frac{1}{2} d C_{n r} r+C_{n v} v+V_{o} C_{n \zeta} \zeta\right) \\
\dot{v} & =\frac{1}{2 m} \rho V_{o} S\left(C_{y v} v+V_{o} C_{y \zeta} \zeta\right)-U r
\end{aligned}
$$

where the body angular velocties are $(r, q)(\mathrm{rad} / \mathrm{sec})$ and the lateral translational velocities are $(v, w)$ (m/sec).

Lateral control of the missile is usually accomplished by controlling either the lateral acceleration or the lateral velocity. For this paper, the control of the lateral velocity will be considered as it does not have the same deficiency in relative degree that control of the lateral acceleration exhibits [2]. The lateral velocity in equation (3) can be expanded using the parametric relationships detailed in [2], to give:

$$
\dot{v}=V^{o}\left(C_{y v} v+V_{o} C_{y \zeta} \zeta\right)-U r
$$

$$
\begin{aligned}
= & V^{o}\left[\left(C_{y v_{0}}+C_{y v_{M}} M+C_{y v_{o}}|\sigma|\right) v\right. \\
& \left.+V_{o}\left(C_{y \zeta_{0}}+C_{y \zeta_{M}} M+C_{y \zeta_{\sigma}}|\sigma|\right) \zeta\right]-U r \\
= & V^{o}\left[\left(\bar{C}_{y v_{0}} v+\bar{C}_{y v_{\sigma}}|v| v\right.\right. \\
& \left.+V_{o} \bar{C}_{y \zeta_{0}} \zeta+V_{o} \bar{C}_{y \zeta_{\sigma}}|v| \zeta\right]-U r
\end{aligned}
$$

where the aerodynamic coefficients $C_{y v}$ and $C_{y \varsigma}$ are functions of Mach number $M$ and incidence $\sigma$. A state feedback controller has been developed which results in linear, decoupled closed loop input/output behavior, and is given by the nonlinear control law $u=\frac{\nu-\alpha}{\beta}$, derived using the feedback linearisation technique [3]. A specified tracking performance for lateral velocity control is thus cheiveded but the design assumes a nominal model obtained from wind tunnel tests with exact knowledge of aerodynamic coefficients. In practice however, this assumption is not valid as each missile will be close to, but not the same as, the wind tunnel model. The aerodynamical functions will be different from their nominal values by the uncertainties $\Delta f(x)$ and $\Delta g(x)$. The feedback linearisation controller will no longer meet the desired performance specifications and hence will not be robust to these uncertainties. The size of the uncertainties $\Delta f(x)$ and $\Delta g(x)$ can be assessed by scoping the size of the uncertainties in the aerodynamic coefficient $C_{y v}$ and $C_{y \zeta}$, together with the static margin $X_{c p}$. Uncertainty ranges of $\pm 25 \%$ change for $C_{y v}, \pm 15 \%$ for $C_{y z}$, and the most sensitive coefficient, $X_{c p}$, has an uncertainty range of $\pm 1.5 \%$ have been used in this paper as representive uncertainties in the respecive coefficients.

In order to achieve robust performance against uncertainties, a fuzzy logic outer loop controller (trajectory outer loop) suppliments the feedback linearisation controller. Two fuzzy logic trajectory controllers are used in the outer loop for the horizontal $v$, and vertical $w$ channels respectively. In order to capture the uncertainty structure a polytope of models is calculated that represent the ver- 
tex models for the parametric uncertainites in the aerodynamic coefficients. This family is used within a GA optimisation procedure to tune the membership functions and the rules of the fuzzy logic controller.

The feedback linearisation controller designed using the nominal model [3] produces a natural frequency of $w_{n}=50(\mathrm{rad} / \mathrm{sec})$ and a damping factor $\zeta=0.7$ for the closed loop system and a fast $250[\mathrm{rads} / \mathrm{sec}]$ second order linear actuator is also included. The closed loop performance is twice as fast as the open loop airframe, hence will produce a challenging robustness problem.

The fuzzy logic trajectory controller has been designed based on a fuzzy inference engine, as a two input - one output system with five membership functions for each variable. The membership functions' positions and the rule parameters are generated using an evolutionary algorithm.

\section{Multi-objective Optimiza- tion}

The proposed framework maintains a population of fuzzy rule sets with their membership functions and uses the evolutionary algorithm to automatically derive the resulting fuzzy knowledge base. A hybrid real valued/binary chromosome has been used to define each individual fuzzy system. The evolutionary algorithm [4] follows the usual format of ranking, selection, crossover, mutation and evaluation but the real and binary parts of the chromosomes are processed separately. A multi-objective approach was used to identify good solutions. A population of 100 individuals was maintained by the algorithm and at each generation, 20 individuals were selected for breeding. Crossover was performed at a rate of 0.9 , with intermediate crossover being used for the real values and uniform multipoint crossover for the binary part. A mutation rate of $2 / 137$ was used. Selective pressure (SP) of 1.7 is used. The high crossover and low selective pressure is designed to slow convergence in order to prevent local optimum being selected. The twenty new individuals were evaluated and then concatenated to the old population, forming a set of 120 individuals. Non-dominated ranking was then applied to this set and the best 100 were taken for the next generation.

The non-dominated ranking method was used in the evolutionary algorithm to allow the multiobjective problem to be handled easily. A detailed description of the non-dominated ranking process may be found in [5], and is based on several layers of classifications of the individuals. To classify the individuals, the population is ranked on the basis of non-domination: all non dominated individuals are classified into one category (with a dummy fitness value, which is proportional to the population size, to provide an equal reproductive potential for these individuals). To maintain the diversity of the population, these classified individuals are shared using their dummy fitness values. Each category of non dominated individuals is considered in turn and the process continues until all individuals in the population are classified.

The evolutionary algorithm behavior in terms of convergence and searching through feasible regions for acceptable solutions will depend on how the multiple objectives are used. In a previous paper [1] we have used the surrogate additive function which transforms the vectorised multi-objective problem into a scalar optimisation problem. In this paper we have defined the closed loop performance criterias as four competing objectives by using the reference point approach [6]. Other researchers [7] have applied similar ideas to a gas turbine engine model.

Competing objectives force a trade-off solution to be sought. The tradeoff information generated by the evolutionary algorithm can contribute to a better understanding of the complexity of the problem. Generally, the objective criteria are not directly comparable and their numerical values may differ considerably. This can make the tradeoff strategies difficult to define. A procedure for normalisation has been used to convert the criteria $y_{j}(\mathbf{x})$ into a dimensionless function $\eta_{j}(\mathbf{x}) \in[0, \mathbf{1}]$ has been used to make the trade-off problem easier. One such normalisa5tion procedure is the optimistic reference point approach [6] which redefines the objectives relative to an ideal values $y_{j}^{\star}$ :

$$
\eta_{j}(\mathbf{x})=\frac{\mathbf{y}_{\mathbf{j}}^{\star}-\mathbf{y}_{\mathbf{j}}(\mathbf{x})}{\mathbf{y}_{\mathbf{j}}^{\star}}, \mathbf{j} \in[\mathbf{1}, \ldots, \mathbf{m}]
$$

If the ideal value $y_{j}^{\star}$ is small or $y_{j}^{\star} \rightarrow 0$, an alternative form can be used:

$$
\eta_{j}(x)=\frac{\mathbf{y}_{\mathbf{j}}^{*}-\mathbf{y}_{\mathbf{j}}(\mathbf{x})}{\mathbf{y}_{\mathbf{j} \max }-\mathbf{y}_{\mathbf{j} \min }}, \mathbf{j} \in[\mathbf{1}, \ldots, \mathbf{m}]
$$

where $y_{j \max }$ and $y_{j \min }$ are respectively the maximum and minimum values of the criterion $y_{j}(\mathbf{x})$ in $\mathbf{x}$. The optimistic reference point method is applied to all four closed loop performance criteria: rise time, steady state error, overshoot and settling time, defined as follows: 
- Side-slip velocity steady state error:

$$
\eta_{j}(x)=\frac{E r_{j}^{\star}-E r_{j}(\mathbf{x})}{E r_{j \max }-E r_{j \min }}, j \in[1, \ldots, m] .
$$

- Overshoot:

$$
\eta_{j}(x)=\frac{O s_{j}^{\star}-O s_{j}(\mathbf{x})}{O s_{j \max }-O s_{j \min }}, j \in[1, \ldots, m] .
$$

- Rise time:

$$
\eta_{j}(x)=\frac{T r_{j}^{\star}-T r_{j}(\mathbf{x})}{T r_{j \max }-T r_{j \min }}, j \in[1, \ldots, m] .
$$

- Settling time:

$$
\eta_{j}(x)=\frac{T s_{j}^{\star}-T s_{j}(\mathbf{x})}{T s_{j \max }-T s_{j \min }}, j \in[1, \ldots, m] .
$$

Table 1 defines the reference point parameters.

\section{Fuzzy gain scheduled con- trollers for multiple de- mands}

The fuzzy controllers for the horizontal and vertical channels are shown in figure 1 . The inputs to the controller consist of the side-slip velicity error and its derivative, and the output is the input to the feedback linearisation control loops. Each input variable is covered using 5 membership functions.

The problem of finding a single set of rules for the polytope of models is compounded by the fact that the nonlinear aerodynamics produce a large range in model characteristics. It is not possible for a single controller to satifactorily control all models to the required specification. In order to solve this problem, a scaling factor that is a function of the parametric variables is considered. The greatest variation is seen in the change of incidence $\sigma$. For a contstant forward speed $V_{o}$ this translates into a side-slip velocity dependency. ' 1 he fuzzy logic controller inputs and outputs are thus scaled by a scaling factor, and the rule parameters are treated as constants to be determined by the evolutionary algorithm. This concept has been used by other workers [8] to improve the performance and stability of fuzzy controllers. In Bonissone's chapter@[8], scaling factors of an FLC have been tuned by GA's. Some time scaling factors are used to fine tune the performance of the system in a similar way to the tuning of a PID controller. In [9] the firing of the rules in a fuzzy controller is described with different values for the scaling factors. This demonstrates the fact that the adjustment of the factors is equivalent to the re-construction of the membership functions in the rule-base.

The three scaling factors (error,derivative of error and output) for each required lateral acceleration demand $1 g, 2 g, \ldots, 15 g$ have been determined via steady state analysis of the model polytope using the fixed gain trajectory controller around the nominal model. Then a polynomial fitting has then been evaluated to interpolate between the specific velocity demands. The scaling factor for the velocity error is $1^{s t}$ order:

$$
S C_{v-e r}=f\left(v_{d}\right)=v_{d}
$$

and the polynomial curve for the velocity error scaling factor is shown in figure 2. The scaling factor for

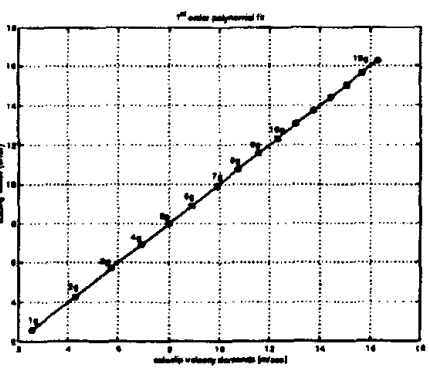

Figure 2. $S C_{v-e r}=f\left(v_{d}\right)=v_{d}$

the derivative of error is of a $3^{\text {rd }}$ order polynomial:

$$
S C_{v-\text { erd }}=f\left(v_{d}\right)=p_{3} v_{d}^{3}+p_{2} v_{d}^{2}+p_{1} v_{d}+p_{0}
$$

and the output scaling factor is of $1^{\text {st }}$ order polynomial.

$$
S C_{\text {out }}=f\left(v_{d}\right)=q_{1} v_{d}+q_{0}
$$

where $p_{0}, \ldots, p_{4}$ and $q_{0}, q_{1}$ are the polynomial fit coefficients for each scaling factors respectively.

\section{Results}

The optimization procedure produces multiple solutions and has been able to tune the membership function parameters simultaneously for multiple demands. In a typical run, about $90 \%$ of the solutions in the final population are non-dominated. Figure ?? shows a detailed trade-off for the nondominated solutions at the last generation. Two alternative solutions have been presented in figure 6 to show the effect of the interpolative mechanism of 


\begin{tabular}{|l|l|l|l|l|}
\hline Reference points & Steady State Error & Settling time & Rising time & Overshoot \\
\hline Ideal point & $E r_{j}^{\star}=0.0(\%)$ & $T s_{j}^{\star}=0.15(\mathrm{sec})$ & $T r_{j}^{\star}=0.08(\mathrm{sec})$ & $O s_{j}^{\star}=4.5(\%)$ \\
\hline Maximum & $E r_{j \max }=2.0(\%)$ & $T s_{j \max }=0.25(\mathrm{sec})$ & $T r_{j \max }=0.14(\mathrm{sec})$ & $O s_{j \max }=25.0(\%)$ \\
\hline Minimum & $E r_{j \min }=0.0(\%)$ & $T s_{j \min }=0.1(\mathrm{sec})$ & $T r_{j \min }=0.07(\mathrm{sec})$ & $O s_{j \min }=2.0(\%)$ \\
\hline
\end{tabular}

Table 1. Closed loop performance criteria

the fuzzy scheduled controllers when multiple demands are used. Solution (b) has very little overshoot, almost no steady state error and good settling time. Solution (c) has a very slow rise time, very little overshoot, a long settling settling time but a good steady state error. Solution (b) is chosen as the final controller. On the left side of figure $6(a)$ is the nonlinear control surface found by the GA's of the FLC.
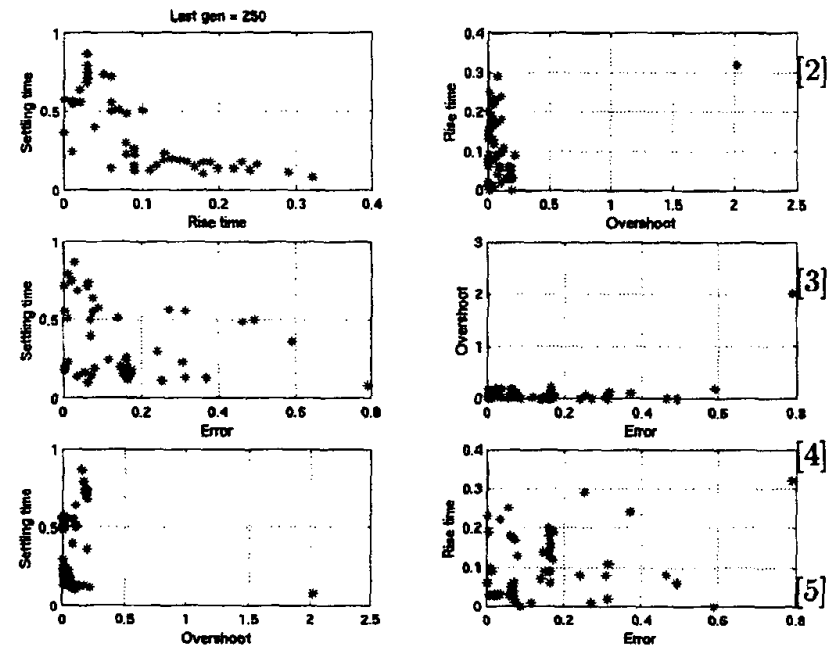

Figure 3. Trade-off between each objectives at last gen 250

\section{Conclusions}

Evolutionary algorithms have been successfully applied to a multimodal search space in the context of determining the control parameters for the missile autopilot. The optimization has used multiple objectives related to closed loop performance: steady state error, overshoot, settling and rise time. Multiple solutions have been obtained using a Pareto based approach combined with a reference point approach to incorporate preference information into the GA to direct the search towards feasible desired area from which the designer can choose solutions that satrisfy the performance specifications in the face os parametric uncertainty. The design has been exercised for a large range of velocity demands $(1 \mathrm{~g}, 5 \mathrm{~g}, 10 \mathrm{~g}, 15 \mathrm{~g}$-lateral acceleration equivalent).

\section{References}

[1] E. H. A. Blumel, B. A. White, "Design of robust fuzzy controllers for aerospace applications," in Proceedings of the 18 NAFIPS Conference, vol. 1, pp. 438-442, 1998.

[2] B. A. White, "Non-linear autopilot of the horton and generic missile," tech. rep., Dept. of Aerospace, Power and Sensors, Cranfield University-RMCS, Shrivenham, Wilts, SN6 8LA, October 151998.

[3] A. L. B. A. Tsourdos and B. A. White, "Trajectory control of a nonlinear homing missile," in Proc. of the $14^{\text {th }}$ IFAC Symp. on Aut. Control in Aerospace, 1998. Korea.

4] A. Zalzala and P. J. Fleming, eds., Genetic algorithms in engineering systems. The Institution of Electrical Engineers, 1997.

5] N. Srinivas and K. Deb, "Multiobjective optimization using nondominated sorting in genetic algorithms," Evolutionary Computation, vol. 2, no. 3, pp. 221-248, 1995.

[6] H. Weistroffer, "A combined over and underachievement programming approach to multiple objectives decision making," Journal of Large Scale Systems, vol. 7, pp. 47-58, May 1984.

[7] C. M. Fonseca and P. Fleming, "Multiobjective optimization and multiple constraint handling with evolutionary algorithms - part 2: Application example," IEEE Thansactions on Systems,Man and Cybernetics, vol. 28, pp. 38-47, January 1998.

[8] C. C. Bonivento and R.Rovatti, eds., Fuzzy Logic Control Advances in Methodology. World Scientific Publishing Co, 1998.

[9] Y.-Y. Chen and C. Perng, "Input scaling factors in fuzzy control systems," in Proc. IEEE, pp. 1666-1670, 1994. 


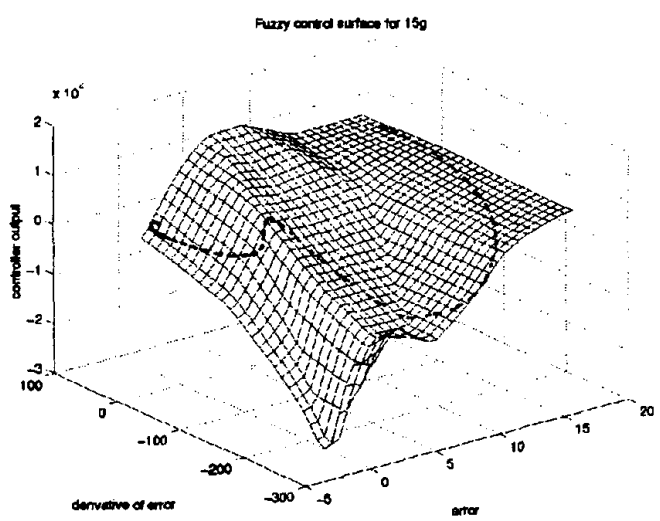

a) Fuzzy control surface for $15 \mathrm{~g}\left[150 \mathrm{~m} / \mathrm{sec}^{2}\right]$
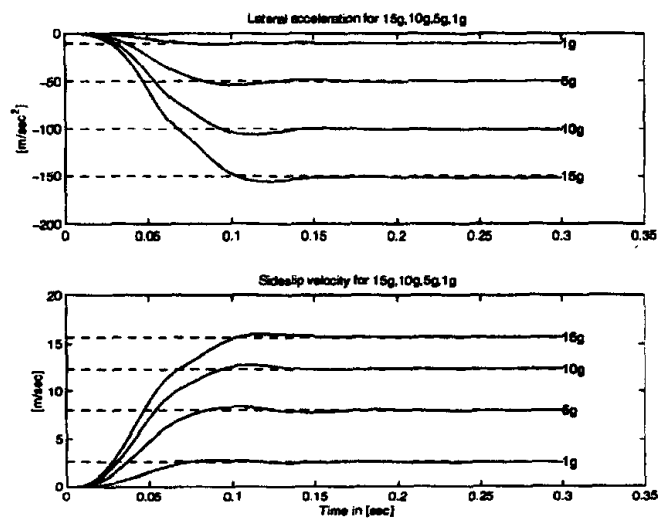

b) Best Solution for steady state error
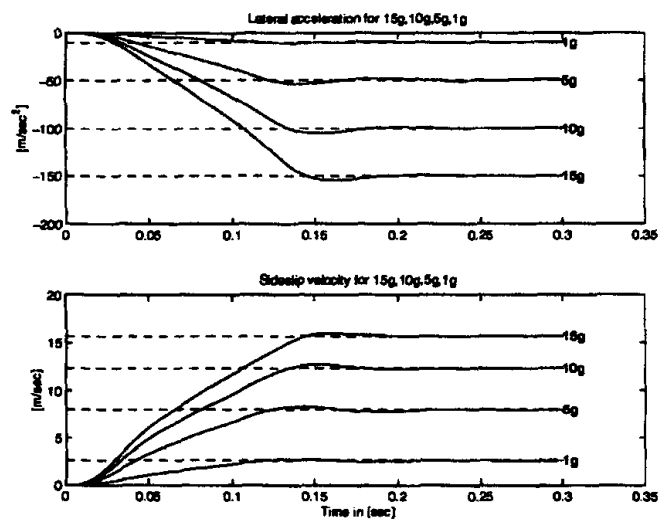

c) Slow rise time solution
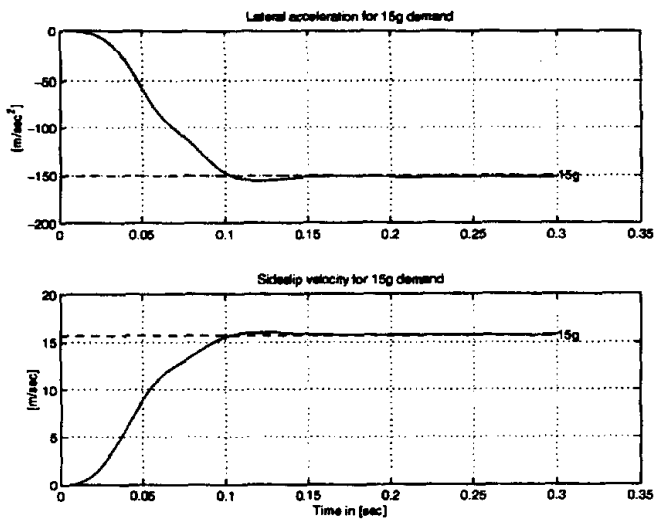

Side-slip velocity and lateral acceleration for $15 \mathrm{~g}$

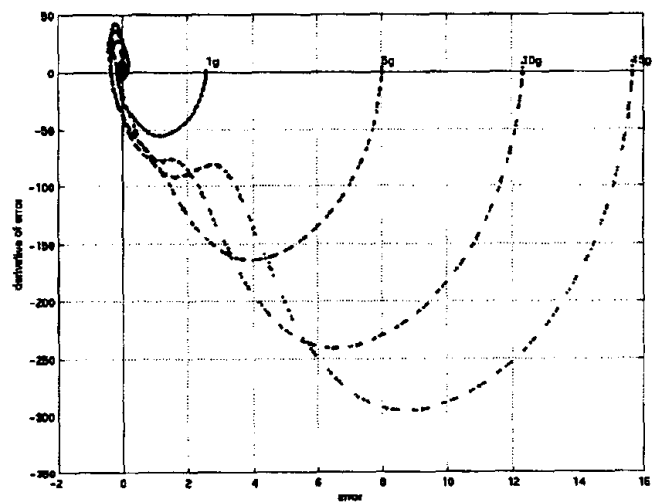

Phase portrait for steady state solution

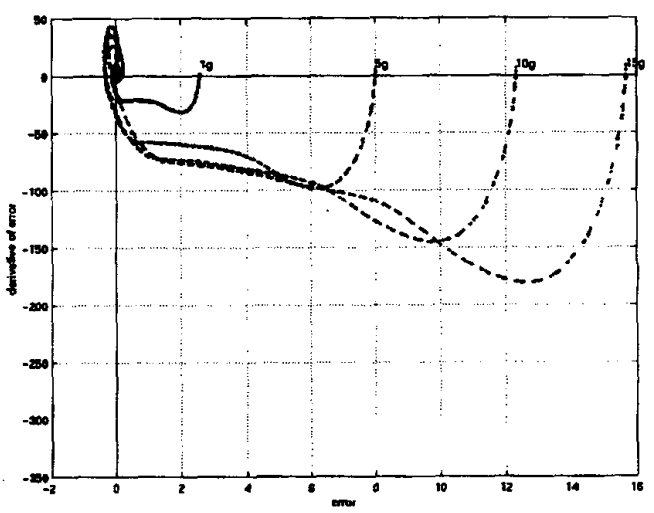

Slow solution phase portrait

Figure 4. Multiple solutions for multiple demands 